\title{
Measurement of the low energy (20-300 MeV) electron and positron spectra with the AESOP-Lite balloon mission
}

\author{
S. Mechbal ${ }^{* a}$, J. Clem $^{b}$, P. Evenson ${ }^{b}$, R. Johnson ${ }^{a}$, B. Lucas ${ }^{b}$, P.-S. Mangeard ${ }^{b}$, J. \\ Roth $^{b}$ \\ ${ }^{a}$ Santa Cruz Institute for Particle Physics, Physics Department, University of California Santa \\ Cruz, Santa Cruz, CA 95064, USA. \\ ${ }^{b}$ Bartol Research Institute, University of Delaware, Newark, DE 19716, USA. \\ E-mail: smechbal@ucsc.edu, clem@bartol.udel.edu, evenson@udel.edu, \\ rjohnson@ucsc . edu, lucasb@udel . edu, mangeard@udel . edu, \\ roth@bartol.udel.edu
}

We report on a measurement made of cosmic ray electrons and positrons between 20-300 MeV with the balloon-borne spectrometer AESOP-Lite, which was flown from Esrange, Sweden to Ellesmere Island, Canada in May 2018, at an average altitude of $\sim 3 \mathrm{~g} \mathrm{~cm}^{-2}$ of residual atmosphere. The maiden AESOP-Lite flight took place in May 2018, accumulating roughly 130 hours of exposure above 130,000 feet. This paper gives an overview of AESOP-Lite's science goals, instrumentation and the 2018 balloon campaign. We describe the selection criteria for particle identification, the methodology of the analysis, and very preliminary results of the electron spectrum in the energy regime of 20 to $300 \mathrm{MeV}$ obtained on this flight.

36th International Cosmic Ray Conference -ICRC2019-

July 24th - August 1st, 2019

Madison, WI, U.S.A.

\footnotetext{
${ }^{*}$ Speaker.
} 


\section{Introduction}

Despite the great progress accomplished in the past decade in precision measurements of cosmic rays with space-borne instruments (PAMELA, AMS-02), a gap in the knowledge of the electron and positron spectra below $100 \mathrm{MeV}$ remains. In this energy range, the effects of the solar modulation are the most influential, and can be compared to the unmodulated VLIS (Very Local Interstellar Spectrum) provided by Voyager. The negative spectral index below $100 \mathrm{MeV}$ (see Figure 1) has been observed since the 1960's, yet the mystery of its origin persists: it is then necessary to make simultaneous measurements of electrons and positrons in order to reach any further conclusions. The charge sign of cosmic ray particles can affect their propagation. Careful study of particle and antiparticle species allows for a definitive separation of the effects due to charge sign from the other possible factors. Furthermore, the positron fraction has never been resolved below $100 \mathrm{MeV}$, whereas PAMELA [1], Fermi-LAT[2], AMS-02 [3] reported a fraction higher than predicted from a purely secondary model above $1 \mathrm{GeV}$.

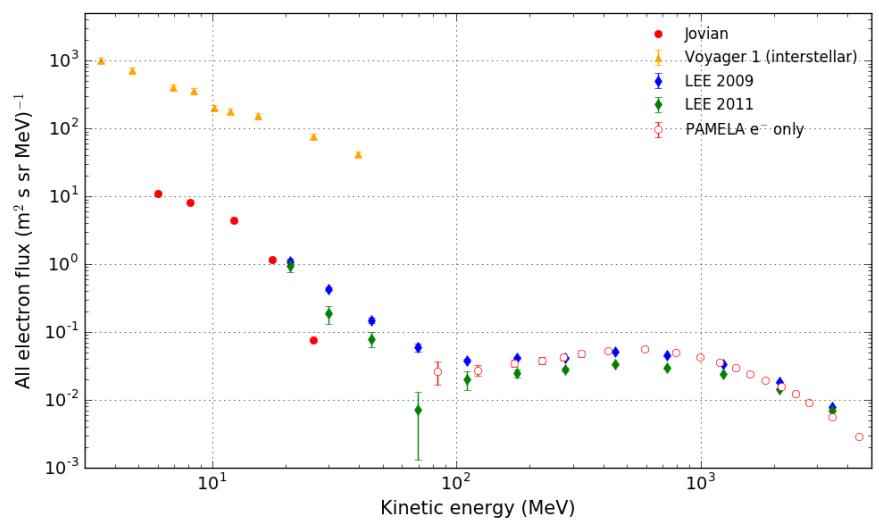

Figure 1: The electron spectra from previous measurements (see $[4,5,6,7])$. Observe the turn-up that occurs at around $100 \mathrm{MeV}$.

\section{AESOP-Lite instrument}

The AESOP-Lite instrument is a balloon-borne compact magnetic spectrometer designed to measure primary electrons and positrons between 20-300 MeV. It is the successor to the LEE (Low Energy Electrons) payload [8], which retired after 23 successful flights, providing important measurements of low-energy electrons. The instrument has been modified by replacing the calorimeter with a magnetic spectrometer, making it possible to resolve the charge-sign. The original entry telescope has been kept however: it consists of three plastic scintillators and a Cherenkov gas detector (for a detailed summary of the instrument and its performance, see [9]). The tracking system consists of 7 planes of silicon strip detectors (SSDs) and a Halbach ring dipole magnet. The average field is $0.3 \mathrm{~T}$, and the detectors are placed in an $x y$-configuration, with 4 layers in the bending plane to measure the particle deflection, and 3 layers to view their trajectory in the non-bending plane. The momentum resolution is $\sim 12 \%$ with an overall geometry acceptance of $\sim 15 \mathrm{~cm}^{2} \mathrm{sr}$ at $300 \mathrm{MeV}$.

\section{2018 campaign}

\subsection{The flight}

AESOP-Lite launched on May 15th 2018 from Esrange, Sweden $\left(67^{\circ} 89^{\prime} \mathrm{N}\right)$ on a NASA 40 

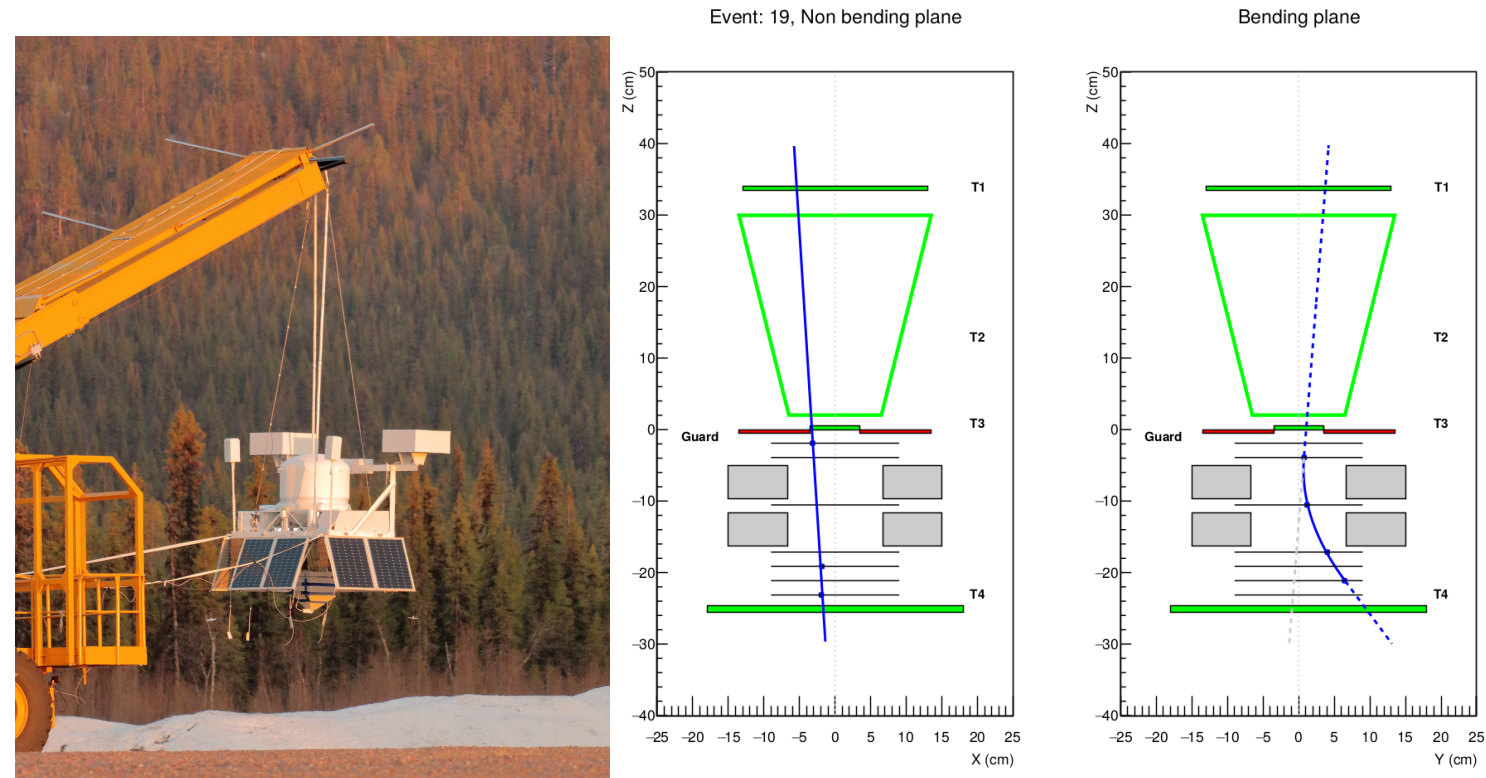

Figure 2: (Left) Picture of the integrated AESOP-Lite payload at Esrange on the flight line. (Right) Schematic of instrument seen in the event display, for a $25 \mathrm{MeV}$ electron event.

MCF (Million Cubic Feet) zero pressure long duration stratospheric balloon for a 133 hour-long flight at an average altitude of $135 \mathrm{kft}\left(\sim 41 \mathrm{~km}\right.$, which corresponds to $\sim 3 \mathrm{~g} \mathrm{~cm}^{-2}$ atmospheric overburden). It landed on Ellesmere Island, Canada $\left(78^{\circ} 40^{\prime} \mathrm{N}\right)$. The northerly trajectory of the payload allowed the experiment to survey regions of low rigidity cutoff (below $200 \mathrm{MV}$ ), as illustrated by the flight map in the right panel of Figure 3.

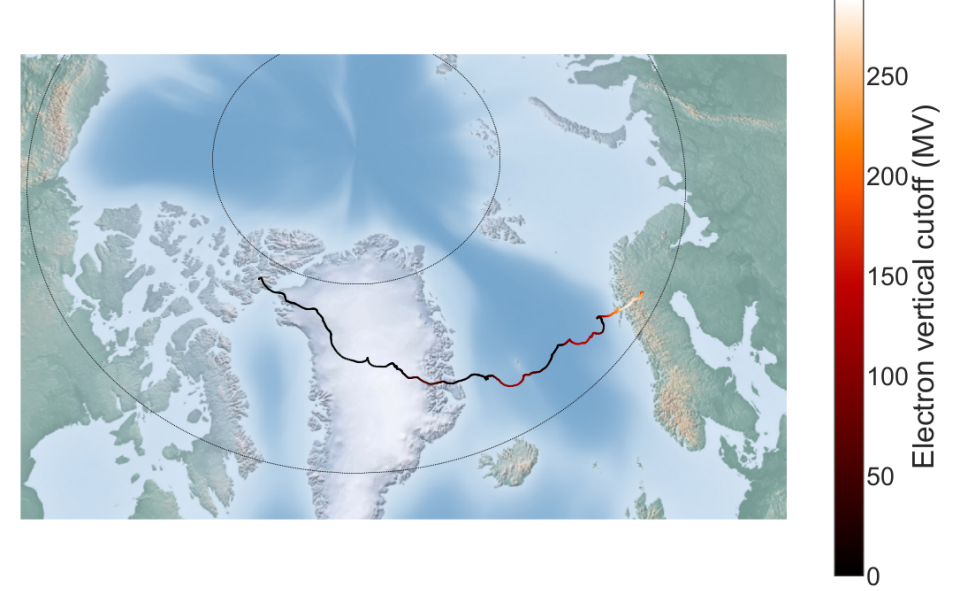

Figure 3: Trajectory of the first flight. The first 90 hours of the flight surveyed latitudes where diurnal variations of the geomagnetic field are still present, as indicated by the color-coded legend.

In its flight configuration, the instrument sits inside a $2.5 \mathrm{~cm}$ thick pressure vessel made of aluminum with a polyurethane foam insulation (Figure 2, left panel). The total weight of the gondola, including the batteries and solar panels, is $940 \mathrm{lbs}$, and the payload launched with an additional $600 \mathrm{lbs}$ of ballast. Four $100 \mathrm{~W}$ solar panels provide power to the instrument in flight, which uses $73 \mathrm{~W}$. Three telemetry channels are used for monitoring the good health of the apparatus and receiving data in real time: line-of-sight, low-rate Iridium and high-rate Iridium Pilot. The Iridium channels are doubly redundant. Two serial data loggers installed on the gondola, as well as an SD card plugged into the electronic crates inside the shell, were installed to safely record all 
data.

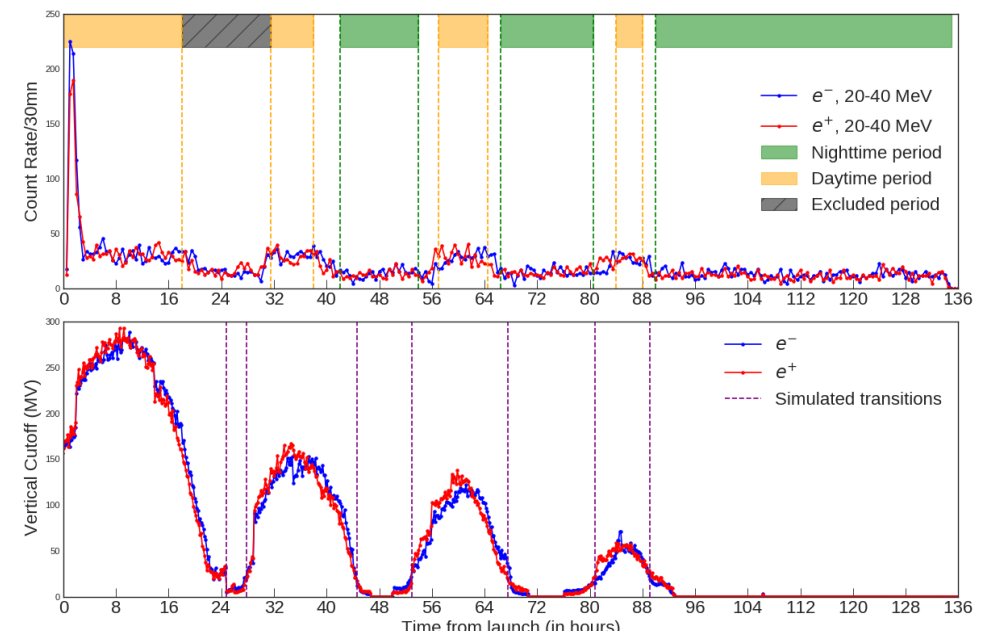

Figure 4: Top Panel: A time series of reconstructed electrons and positrons at the lowest energy bins. The diurnal variations between geomagnetic day and night are clearly visible. The rate of particles rises during geomagnetic day, when secondary albedo particles, produced in the interaction of primary cosmic rays with atmospheric nuclei, are trapped and travel along the geomagnetic field lines. Bottom Panel: Simulated variation of the vertical geomagnetic cutoff using the method of [10].

\subsection{Particle Identification}

The coincidence signal T1-T2-T3 was used as the main trigger during flight. Several additional cuts were made to select good electron and positron events. The first criterion is to select events which occurred during geomagnetic night, when the geomagnetic cutoff is essentially null. This removes the secondary albedo particle component present during geomagnetic day, while keeping events from the first 2.5 hours of the flight, during the ascent. We then require for the guard (anti-coincidence signal) not to have been triggered, to eliminate events that have produced a shower in the instrument. For similar reasons, we also limit the number of hits in the tracker to 5 to 12 hits. To eliminate the scintillation signal from low energy protons in the Cherenkov detector, we apply a cut above the first two photoelectron peaks. Similarly, T2 vetoes muons below $1.5 \mathrm{GeV}$ which are present during the ascent. Muons above that momentum threshold will be reconstructed, but their energy will be well above the maximum detectable rigidity of the spectrometer, so that the cut on the reconstructed momentum should efficiently eliminate them from the signal. A cut on scintillator T3 is applied to discriminate against alpha particles (Figure 5) as the energy deposited grows proportionally to $Z^{2}$. This cut on $\mathrm{T} 3$ incurs a loss of $\sim 5 \%$ of the electron and positron signal.

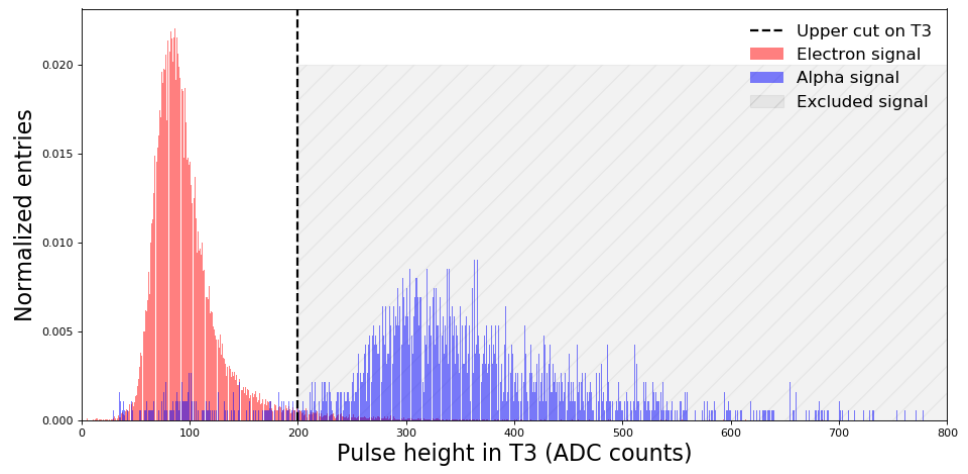

Figure 5: The normalized signal in scintillator T3. A cut is applied at an ADC count of 200, to eliminate the alpha particles above the Cherenkov threshold above that signal.

\subsection{Altitude profile of the measured count rates}

Once electron and positron candidate events have passed the particle identification criteria, the data set is then organized in 7 momentum bins and 14 atmospheric pressure bins. The live time the 
payload has spent in each pressure bins during the flight is measured accordingly. This allows us to produce growth curves for each energy bin, that is, a profile of the count rate of particles as a function of the atmospheric depth. The first 2.5 hours are used to obtain the points during the ascent, where the low energy cosmic ray electron and positron signal is assumed to be purely secondary. Figure 6 presents the growth curves for 2 ranges of momentum for the flight of 2018, respectively $30-50 \mathrm{MeV} \mathrm{c}^{-1}$ (left) and 300-500 $\mathrm{MeV} \mathrm{c}^{-1}$ (right). The ascent and the first 17 hours of the flight occurred during geomagnetic day time. The corresponding data are presented with diamonds in the figure. The full night-time data set is used for the open circles markers and presents the measured count rates free of trapped secondary particles. As seen in the bottom panel of Figure 4, the vertical geomagnetic cutoff varies within the range 0-300 MV during the first daytime period such that we expect to observe a much larger contribution of albedo secondary particles at low energy (below the cutoff) than at higher energies closer to the cutoff $(\sim 300 \mathrm{MV})$. This is indeed the case in the data presented in Figure 6, where the difference between the night time and day time count rate is significantly larger in the left panel $\left(30-50 \mathrm{MeV} \mathrm{c}^{-1}\right)$ than in the right one $\left(300-500 \mathrm{MeV} \mathrm{c}^{-1}\right)$ at float altitude.
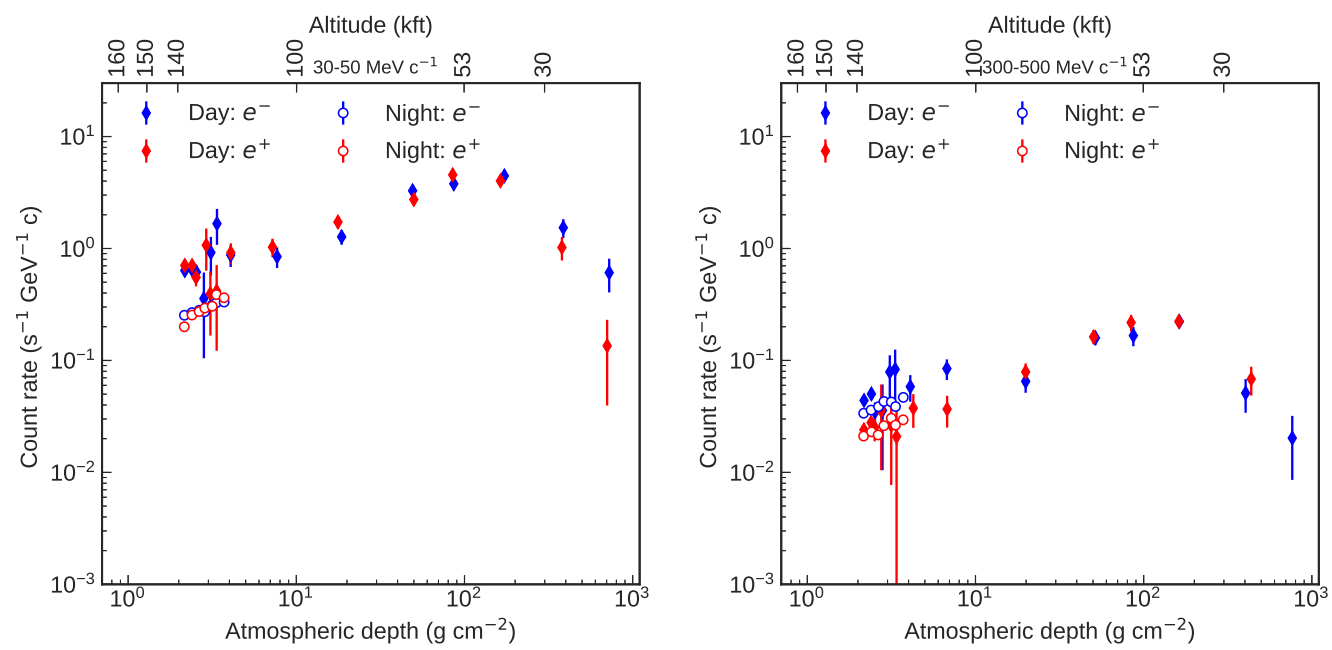

Figure 6: Ascent and night time growth curves for two ranges of momentum.

\section{Analysis Methodology}

During the 5 days of data taking, the balloon floated at an atmospheric depth between 2 and $4 \mathrm{~g} \mathrm{~cm}^{-2}$. In total, the instrument recorded 70 hours worth of data during geomagnetic night. At that altitude, the greatest challenge to obtain a flux at the top of the atmosphere becomes the estimation of the background of electrons and positrons produced in the spallation of Galactic Cosmic Rays (GCR), mostly $\mathrm{H}$ and $\mathrm{He}$, with the nuclei of the residual atmosphere.

To predict this effect, we performed Monte Carlo simulations using the software FLUKA 2011 [11, 12]. The method is composed of three stages:

(i) The air shower development induced by $\mathrm{H}$ and $\mathrm{He}$, as well as primary electrons and positrons, is simulated. Secondary particles fluxes are extracted at 19 atmospheric depths from 998 to $0.87 \mathrm{~g} \mathrm{~cm}^{-2}$. 
(ii) The detector response is determined for six types of secondary particles with an independent simulation. Angle-dependent geometry factors, resolution, bias, and efficiency of the momentum reconstruction for electrons and positrons are presented in [9].

(iii) Both simulations' outputs are combined to estimate the detector response to primary cosmic rays, yield functions, and count rates. We note that for the current analysis, only secondary electrons and positrons are taken into account.

The Monte Carlo (MC) atmospheric simulation uses a 3D profile of the atmosphere at Esrange, Sweden taken from the day of launch, following the method of [13]. The injected spectra are normalized to $\mathrm{H}$ and $\mathrm{He}$ local interstellar fluxes derived by [14]. The heavy nuclei are assumed to produce showers similar to those from $\mathrm{He}$, and are taken into account by applying a scale factor $F_{h n}=1.445$ to the He spectrum [15]. We apply a force-field approximation of the solar modulation [16], using a parameter $\phi=300 \mathrm{MV}$ as calculated from neutron monitor data at the time of the flight, using the method of [15]. Uncertainty on this parameter is $\sim 50 \mathrm{MV}$. Systematic effects using $250 \mathrm{MV}$ and $350 \mathrm{MV}$ were found to be negligible compared to the statistical uncertainties of the results presented in this paper. Due to the acceptance of the entry telescope, the secondary particles with an incident angle $\theta>40^{\circ}$ are not considered. The fluxes of the most vertical incident particles are calculated separately for the intervals $0.9<\cos \theta \leq 1$ and $\cos ^{-1} 40^{\circ} \leq \cos \theta<0.9$. Our flight data are arranged as count rates in units of $\mathrm{s}^{-1} \mathrm{GeV}^{-1} \mathrm{c}$. In order to replicate these rates with our simulation results, we must take into account the instrument's overall response, by applying the geometry factor and the momentum reconstruction probability density functions to the MC flux. The acceptance and the resolution, bias, and efficiency of the reconstruction, pre-detection energy losses are all included.
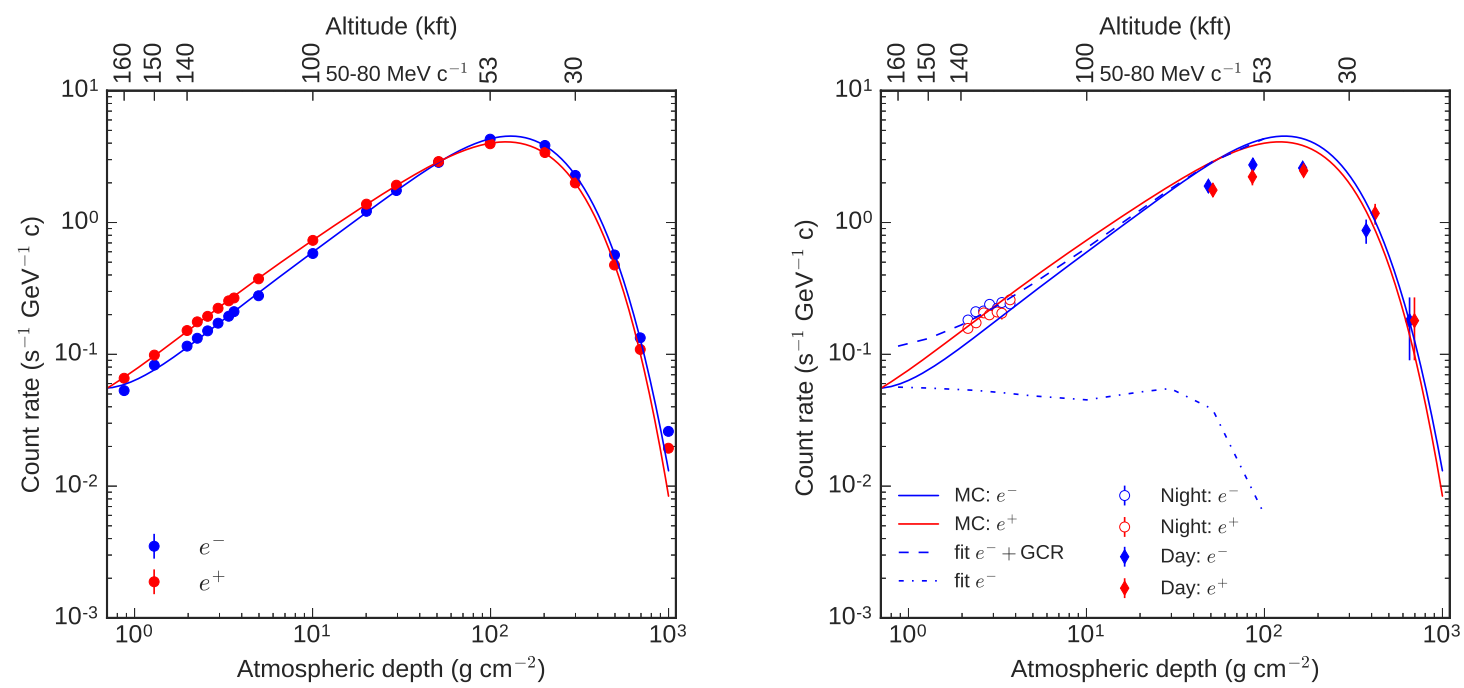

Figure 7: (Left) Monte Carlo growth curves for positron and electrons for the momentum range $50-80 \mathrm{MeV} \mathrm{c}^{-1}$. (Right) Growth curves of flight data and Monte Carlo estimates. The dash line represents the sum of the solid blue line (contribution of secondary electrons) and the dash-dotted line (contribution of primary electrons).

The left panel of Figure 7 shows the simulated growth curves of $\mathrm{e}^{-}$and $\mathrm{e}^{+}$for one momentum bin. The solid line represents the fit to a $7^{\text {th }}$ degree log polynomial function. We are following the philosophy behind past analyses of fitting data points to analytically derived growth curves [17] to 
estimate the background contribution to primary cosmic rays at float altitudes.

\section{Discussion}

This analysis method of our background-dominated signal presents us with the delicate challenge of cross-checking and scaling the simulated growth curves. As previously mentioned, we assume all $\mathrm{e}^{-}$and $\mathrm{e}^{+}$to be of purely secondary origin during the ascent through the atmosphere. The shape of the data and MC growth should then be similar, and the question remains whether and how to normalize the MC to our curve. The fast ascent also made it difficult to get high statistics at the lowest altitude point. We have found that normalizing the simulated growth curves to the observed count rate at Pfotzer maximum (75-450 g.cm ${ }^{-2}$ ) led to an apparent underestimation of the background at float altitude and an overestimation of the spectrum at the top of the atmosphere.

We must keep in mind that the development of the cosmic ray air shower does vary between simulation packages ([18]), such that there are systematic uncertainties concomitant with the use of a single MC software package. However, these systematic uncertainties are expected to be lower at higher altitude, where the atmospheric overburden is less. We have calculated the spectrum of secondary electrons using no normalization of the MC. We observe in the right panel of Figure 7 that the MC overestimates the spectrum at the Pfotzer maximum, and at float altitude it predicts a positron background consistent with the level of the measured signal. More work will be needed on understanding the normalization of the MC prediction of the flux of secondaries of both species before we can present a measurement of the flux of primary positrons. At this preliminary stage, we have derived the flux of electrons only at the top of the atmosphere. The results are presented in Figure 8 . These very early results indicate that the negative slope below $100 \mathrm{MeV}$ remains during the period of positive solar polarity. The spectrum is quite similar to previous measurements at the same overall modulation level (2009).

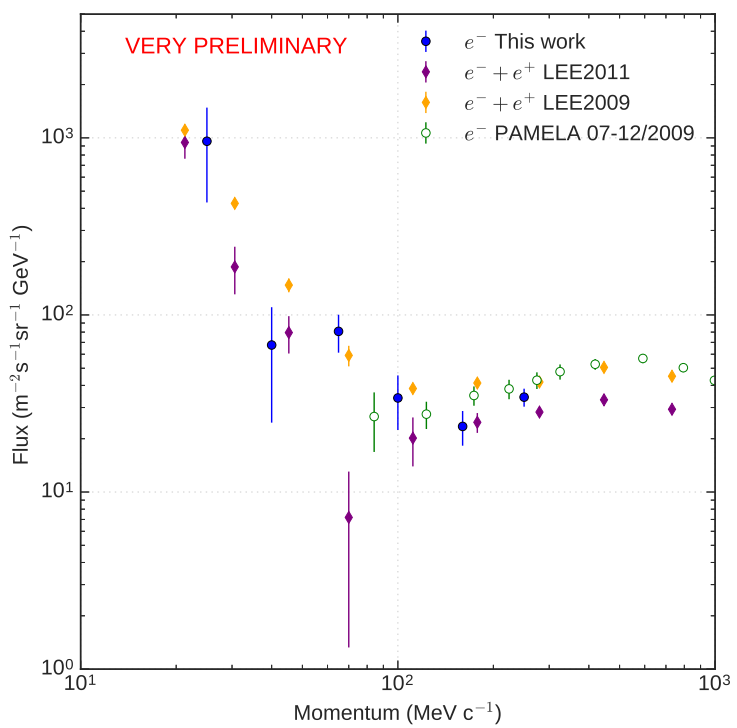

Figure 8: The preliminary AESOP-Lite electron spectrum. The quoted errors are statistical.

Resolving the issue of background reduction is the central topic of investigation before we can produce a definitive spectrum and resolve the positron signal. More work is needed to understand 
the charge-sign dependence of particle species to the heliospheric magnetic polarity at this energy range. The ultimate resolution of these issues would be much easier with a higher altitude flight. This is now technically feasible, with a $60 \mathrm{MCF}$ balloon which can reach an altitude of 160,000 feet, and we are discussing options with NASA.

\section{Acknowledgement}

We would like to thank CSBF, in particular Chris Field, and the Esrange Space Center for the successful execution of the balloon flight and support during the integration period in Palestine, Texas. This work is supported by NASA Heliophysics Research Division, HTIDES Grant Number 80NSSC19K0746.

\section{References}

[1] Adriani, O. et al., 2009, Nature, 458, 607

[2] The Fermi LAT Collaboration, 2018, PRL, 108

[3] Aguilar, M. and others AMS Collaboration, 2013, PRL, 110, 141102

[4] Adriani, O. et al, 2015, ApJ, 810, 142

[5] Evenson, P., Clem J, Proceedings of ICRC 2011, 11, 52

[6] Cummings, A. C. et al. 2016, ApJ, 831, 18

[7] Moses D., ApJ, 313, 471

[8] Hovestadt, D., Meyer, P., Schmidt, P. J., 1970, Nuclear Instruments and Methods, 85, 93

[9] Mangeard, P.-S. et al, 2019, PoS ( ICRC2019) 1116

[10] Lin, Z., Bieber, J. W., and Evenson, P., 1995, JGRA, 100, 23, 543

[11] Bohlen, T.T. et al., 2014, Nuclear Data Sheets 120, 211

[12] Ferrari, A., et al., 2005, CERN-2005-10, INFNTC_05/11, SLAC-R-773

[13] Mangeard, P.-S., Ruffolo. D., Saiz, A., et al. 2016, JGRA, 121, 7435

[14] Ghelfi, A., Barao, F., Derome, L., \& Maurin, D. 2016, A\&A, 591, A94

[15] Ghelfi, A., Maurin, D., Cheminet, A., et al. 2017, AdSpR, 60, 833

[16] Gleeson, L. J., Axford, W. I., 1968, ApJ, 154, 1011

[17] Daniel, R.R., and S.A. Stephens, 1974, Rev. Geophys. Space Phys. , Vol. 12, 2, 235

[18] Mishev, A.L, Velinov, P.I.Y, 2014, JASTP, 120, 111 